\title{
"Blind" protected specimen brushing versus bronchoscopic techniques in the aetiolological diagnosis of ventilator- associated pneumonia
}

\author{
S. Bello*, A. Tejada**, E. Chacón*, M.C. Villuendas+, A. Senar*, \\ M. Gascón*, F.J. Suarez++
}

\begin{abstract}
"Blind" protected specimen brushing versus bronchoscopic techniques in the aetiolological diagnosis of ventilator-associated pneumonia. S. Bello, A. Tejada, E. Chacón, M.C. Villuendas, A. Senar, M. Gascón, F.J. Suarez. (CERS Journals Ltd 1996.

ABSTRACT: The aetiological diagnosis of nosocomial pneumonia in intensive care unit (ICU) patients requires a valid, cheap and safe method. This method should be suitable for all mechanically-ventilated patients and all ICUs. The aim of this study was to assess the diagnostic yields of three methods: "blind" bronchial brushing (Accu-Cath)(protective specimen brush-nonbronchofibroscopic (PSB-non BF)); bronchofibroscopic protected specimen brushing (PSB-BF) and bronchoalveolar lavage (BAL).

We prospectively studied the diagnostic values of the three methods as well as the agreement between microbiological results in 74 patients with 88 episodes of clinically suspected ventilator-associated pneumonia (VAP) and 22 control subjects. VAP episodes were also divided into those with $(n=24)$ and without antibiotic pretreatment $(n=64)$, and into those with $(n=78)$ and without $(n=10)$ right lower lobe infiltrates on chest radiography.

No differences were found as regards the bacteriological yield of the three techniques. Furthermore, the rate of concordant results was high; $92 \%$ for PSB-BF and BAL; 84\% for PSB-nonBF and BAL; 85\% for PSB-nonBF and PSB-BF; and 85\% for PSB-nonBF combined with both bronchoscopic techniques. The diagnostic yields in suspected VAP were 66, 59 and $56 \%$ for PSB-nonBF, PSB-BF and BAL, respectively.

We conclude that "blind" bronchial brushing has similar accuracy to bronchoscopic techniques commonly used in the diagnosis of ventilator-associated pneumonia, constituting an interesting alternative in hospitals where fibreoptic bronchoscopy is not available.

Eur Respir J., 1996, 9, 1494-1499.
\end{abstract}

Depts of * Respiratory Medicine, ${ }^{* *}$ Intensive Care Unit, ${ }^{+}$Microbiology. ${ }^{++}$Hospital Clinico, Hospital Miguel Servet, Zaragoza, Spain.

Correspondence: S. Bello Dronda Paseo Sagasta 32-38

50006 Zaragoza

Spain

Keywords: Blind brush catheter nonbronchoscopic bronchial brushing nosocomial pneumonia

protected specimen brush

ventilator-associated pneumonia

Received: December 201994 Accepted after revision January 221996

This study was supported by grant FISS 92/1054.
Inadequate antibiotic treatment is one of the factors that imply a poor prognosis in nosocomial pneumonia. On the other hand, the aetiological diagnosis improves prognosis and reduces the time spent in the intensive care unit (ICU) $[1,2]$. The techniques available for the aetiological diagnosis of pneumonia can be evaluated from three perspectives. Firstly reliability, expressed by sensitivity and specificity. Secondly safety, and thirdly the economic cost and applicability of each technique in a specific hospital. The ideal technique, therefore, would be reliable, safe and inexpensive.

Protected specimen brushing-bronchofibroscopy (PSB$\mathrm{BF}$ ) and bronchoalveolar lavage (BAL) samples obtained with the aid of the bronchoscope have a widely-accepted diagnostic accuracy. However, limitations for their systematic use have been observed. Although bronchoscopy is usually considered as safe, certain drawbacks have been described [3, 4]. Furthermore, it is an expensive technique due to costs in personnel, materials and infrastructure [5]. One further limitation of a practical nature related to the hospital organization and manpower is that bronchoscopy specialists are not available in all hospitals and at all times.

For all these reasons, various authors have sought alternative techniques that would associate the diagnostic accuracy of PSB-BF and BAL with lower costs and a more widespread availability. Studies with the use of "blind" BAL and those that have focused on demonstrating the utility of diverse "blind" bronchial brushing (PSB-nonBF) have been reported [6-8]. ZuCKER et al. [9] first described this type of bronchial brushing in a small infant population. Torres et al. [10] then assessed the usefulness of bronchial brushing through a Métras catheter. MidDLETON et al. [11] first compared the accuracy of PSB-nonBF (Accu-Cath) with PSB-BF, blind endotracheal aspiration, and BAL in 12 patients with ventilator-associated pneumonia (VAP), and found a similar recovery of bateria using these four methods [11]. Recently, Leal-Noval et al. [12] studied 37 cases of pneumonia, and compared the microbiological results obtained by PSB-BF and by a PSB-nonBF (Accu-Cath). Coincident information was obtained in $83.7 \%$; this percentage 
increased to $100 \%$ when pneumonias without right lower lobe involvement were not included in the analysis. Another study, [13], of 40 pneumonia and five control patients, demonstrated that the introduction of a single bronchial brush through the endotracheal tube is not only a simple technique but also yields the same results as PSB-BF in $73 \%$ of patients.

The aim of the present study was to evaluate the accuracy of PSB-nonBF (Accu-Cath ${ }^{\circledR}$ model PCC 215) for the aetiological diagnosis of VAP and its comparison to PSB-BF and BAL.

\section{Materials and methods}

\section{Patients}

A total of 88 clinically suspected VAP episodes were studied in 74 patients. They had been mechanically-ventilated for at least $48 \mathrm{~h}$ prior to the onset of clinical manifestations of VAP. Clinical suspicion of pneumonia was based on criteria described elsewhere [14]. Bronchial sampling was performed in 14 patients more than once, due to suspected recurrence of VAP. The study excluded patients with severe immunodeficiency and those with contraindications for bronchoscopic procedures [14$16]$.

The following information was obtained from the patients' medical records: name, history, findings on clinical examination, file number, age, gender, main cause of ICU admission, antibiotic treatment on the study day, and radiological manifestations.

The mean age of the patients studied was $52 \pm 20 \mathrm{yrs}$; and $77 \%$ of the patients were male. The two most frequent diagnostic groups were: severe head trauma (39\%) and postsurgery (18\%). The other diagnostic groups were: cerebral stroke (12\%); anoxic encephalopathy after cardiac arrest (4\%); chronic obstructive pulmonary disease (COPD) (13\%); and a "miscellaneous" group (13\%). Of the 88 episodes studied, 24 were receiving antibiotic treatment $24 \mathrm{~h}$ prior to sampling; and 64 were not. Right lower lobe involvement was observed in 78 episodes.

A small group of 22 mechanically-ventilated patients with no clinical criteria for VAP and who were not receiving antibiotic treatment was also evaluated.

This study was approved by the Ethics Committee of Hospital Miguel Servet.

\section{Study design}

The three techniques evaluated in this prospective study were: PSB-BF, PSB-nonBF and BAL. The microbiological information obtained was assessed: 1) by comparing the different percentages of significant, nonsignificant and sterile cultures; and 2) by studying the concordant and divergent microbiological information obtained from each of the three techniques. Bronchoscopic or "blind" bronchial brushing were randomly performed. However, PSB-BF was always performed before BAL. Bronchial and blind samples were obtained consecutively and within a 30 min time-period.

\section{Methodology}

Bronchial sampling (PSB-BF, PSB-nonBF and BAL) was performed upon all patients enrolled into the study. Prior to the bronchoscopic procedure, the patient was ventilated with $100 \%$ oxygen for $15-20 \mathrm{~min}$ and later sedated with Midazolam. Pancuronium was also administered as a muscle relaxant. The administration of local anaesthetics was avoided. All patients underwent pulse oximetry monitoring during the bronchofibroscopic procedures. During bronchoscopy, patients were manually ventilated with a Kuhn@ $\odot$ bag through a T-tube placed at the distal end of the endotracheal tube (Superset Catheter Mount with a Swivel Elbow, 15M-15F, TW2 6RS). The bronchial sampling was performed in the area of maximal radiological involvement. In case of diffuse infiltrates, BAL was carried out in the middle lobe or in the lingula. Following a brief inspection of the bronchi through the bronchofibroscope (Olympus Type 20D), sampling was performed with PSB-BF following a technique described previously [17], using a discardable microbiology brush (No. 2.8, Palex, S.A.). BAL was then performed by instilling three $50 \mathrm{~mL}$ aliquots, of $\mathrm{NaCl}$ $0.9 \%$; the first aliquot was discarded as it was considered to be contaminated from bronchial secretions. The samples were immediately (less than $5 \mathrm{~min}$ ) carried to the microbiology laboratory.

The methodology for the PSB-nonBF sampling was as follows: using aseptic techniques, the PSB-nonBF kit was opened (Accu-Cath, Pulmonary Culture Catheter with Cytology Brush, model PCC-215; HML Medical Inc.). The double-sheathed, balloon-tipped, plugged catheter was introduced through the endotracheal tube and was advanced to its most distal position. The inner catheter was slid out of the external sheath and the balloon was everted and filled with $6 \mathrm{~mL}$ of air. The brush was then advanced through the inner sheath; following several expulsion and retraction movements, the brush was left retracted in the channel, the balloon deflated and the entire catheter removed. The distal end of the brush was cut and placed aseptically in the test tube with $1 \mathrm{~mL}$ of Tryptisoil (tryptone-soy bean), as was done with PSBBF. The samples were also sent to the microbiology laboratory in less than $5 \mathrm{~min}$.

\section{Processing of bronchial samples}

"Blind" and bronchoscopic brushing. Upon receipt in the laboratory, the samples were immediately and vigorously shaken into a "vortex"; and then 0.01 and $0.1 \mathrm{~mL}$ aliquots were inoculated into the following agar media: blood, chocolate, and BCYE- $\alpha$. All cultures were incubated at $37^{\circ} \mathrm{C}$ under aerobic and anaerobic conditions and in a $\mathrm{CO}_{2}$-enriched atmosphere. Cultures were evaluated for growth 24 and 48 h later and were discarded, if negative, after 5 days.

Processing of bronchoalveolar lavage. One chocolate agar plate was cultured with $0.01 \mathrm{~mL}$ of the BAL. The rest of the fluid was centrifuged with the supernatant for culture.

Evaluation of the quantitative cultures. From the bacterial growth obtained on the chocolate agar plates $(0.01$ 
and $0.1 \mathrm{~mL}$ of the transport medium from the two bronchial brushings, or $0.01 \mathrm{~mL}$ of the BAL), colony counts were performed. Multiplying this number by either 10 or 100 , the number of growth colonies per cubic centimeter was obtained. The PSB-BF and PSB-nonBF cultures were "significant" if growth was at least 1,000 colony-forming units (cfu) $\cdot \mathrm{mL}^{-1}$, or if obligate pathogens (e.g. Mycobacterium tuberculosis, Legionella pneumophila) were identified. The cut-off point for the BAL was $\geq 10^{4} \mathrm{cfu} \cdot \mathrm{mL}^{-1}$ [15]. Fungal growth was not considered.

\section{Definitions}

Suspected VAP. The diagnosis of clinically suspected VAP was made on clinical grounds. Modified criteria [14] were used along with the appearance of a new and persistent radiological infiltrate or along with changes in the previous radiological pattern. At least two of the following criteria were required: 1) fever $>38^{\circ} \mathrm{C}$; 2) leucocyte count $>12 \times 10^{9}$ cells $\cdot \mathrm{L}^{-1}$; and 3 ) an increase of bronchial secretions or change in their characteristics. Pneumonia was the suspected diagnosis once all other clinical entities were ruled out (e.g. cardiac failure, atelectasis). In all cases, the clinical and radiological findings appeared at least $48 \mathrm{~h}$ after mechanical ventilation.

Absence of suspected VAP. Those patients who did not meet clinical criteria for pneumonia, or if showing any of the criteria, had another proven diagnosis.

Concordant cultures. When the quantitative cultures of the different techniques offered the same information (quantitatively and qualitatively). A positive quantitative culture had more than $10^{3}$ and $10^{4} \mathrm{cfu} \cdot \mathrm{mL}^{-1}$ for the brush and for the BAL procedures, respectively. A negative culture was sterile or had no significant quantitative growth. Besides the same degree of quantitative growth, the same microorganisms had to be identified.

Discordant cultures. When the techniques tested offered contradictory (not coincident) microbiological information in the quantitative growth.

\section{Statistical analysis}

Statview 4.0, 1992 software was used. The number of organisms recovered with PSB-BF, BAL and PSBnonBF was expressed as colony-forming units per millilitre. Quantitative categories analysis was made using the chi-squared test and percentage difference analysis. A p-value less than 0.05 was considered significant.

\section{Results}

Overall results $(n=88)$

Tables 1 and 2 show the results obtained with quantitative cultures. The greatest diagnostic yield was obtained with PSB-nonBF (57\%), followed by PSB-BF $(51 \%)$, and by BAL (48\%). No significant differences were found for the sensitivity rates between the three techniques $\left(\chi^{2}\right.$ $=1.57$ ).
Table 1. - Overall results of microbiology (all three techniques combined) in 88 episodes of clinically suspected ventilator-associated pneumonia

\begin{tabular}{lc}
\hline No growth (no growth or nonsignificant growth) & $34(39 \%)$ \\
Growth (significant growth) & $54(61 \%)$ \\
Monobacterial growth & 43 \\
S. aureus & 8 \\
Ps. aeruginosa & 14 \\
A. calcoaceticus & 7 \\
H. influenzae & 2 \\
S. pneumoniae & 5 \\
Enterobacter spp. & 3 \\
L. pneumophila & 1 \\
S. epidermidis* & 1 \\
Corynebacterium spp.* & 1 \\
E. coli & 1 \\
Polymicrobial growth & 11 \\
H. influenzae + S. aureus $(2)$ & \\
S. viridans + Enterobacter spp. + A. calcoaceticus* & \\
S. aureus + K. pneumoniae + Ps. aeruginosa & \\
S. marcescens + A. calcoaceticus & \\
Ps. aeruginosa + Corynebacterium spp. + S. aureus* & \\
S. epidermidis + Corynebacterium spp. + H. influenzae* & \\
H. influenzae + E. coli + S. aureus & \\
E. coli + P. mirabilis & \\
S. aureus + Ps. aeruginosa &
\end{tabular}

*: patients with severe malnutrition in whom thoracic surgery was performed and improvement was observed after specific antibiotherapy.

The cultures obtained by PSB-BF and BAL agreed in 81 episodes (92\%); PSB-nonBF and BAL in $74(84 \%)$; and the two bronchial brushings in $75(85 \%)$. The microbiological information obtained by PSB-nonBF combined with both bronchoscopic procedures agreed in 75 pneumonias $(85 \%)$.

\section{Results from the non-suspected VAP group $(n=22)$}

With PSB-BF sterile cultures were obtained in 20 procedures (91\%); with BAL in $19(86 \%)$; and with PSBnonBF in $17(77 \%)$. When comparing these findings, no significant differences were found.

Results from episodes with vs without antibiotic treatment

In the subgroup of suspected VAP without antibiotic treatment $(n=64)$, significant growth was observed in 38 with PSB-BF (59\%); in 42 with PSB-nonBF (66\%); and in 36 with BAL (56\%). Very high agreement in the microbiological yield was observed in PSB-BF and BAL, $92 \%$ (58 out of 64); PSB-nonBF and BAL, $84 \%$ (54 out of 64); and PSB-BF and PSB-nonBF, $85 \%$ (54 out of 64). Combining PSB-nonBF with PSB-BF and BAL, the results agreed in $85 \%$ of the pneumonias (54 out of 64 ).

In the subgroup of cases receiving antibiotics $(n=24)$, significant growth was observed in 7 (29\%) with PSB$\mathrm{BF}$, in 8 with PSB-nonBF (33\%), and in 6 with BAL (25\%).

No significant differences were observed between the techniques when the cultures with no growth and with nonsignificant growth were grouped together for analysis $\left(\chi^{2}=0.38\right)$. In this subgroup, PSB-BF agreed with 
Table 2. - Results obtained with quantitative cultures

\begin{tabular}{|c|c|c|c|}
\hline Technique & Significant growth & Nonsignificant growth & No growth \\
\hline \multicolumn{4}{|c|}{ Quantitative growth from all suspected VAP episodes studied $(n=88)$} \\
\hline PSB & $45(51)$ & $3(3)$ & $40(45)$ \\
\hline BAL & $42(48)$ & $7(8)$ & $39(44)$ \\
\hline PSB-nonBF & $50(57)$ & $6(7)$ & $32(36)$ \\
\hline \multicolumn{4}{|c|}{ Quantitative growth from the control group $(n=22)$} \\
\hline PSB & $1(5)$ & $1(5)$ & $20(91)$ \\
\hline BAL & $1(5)$ & $2(9)$ & $19(86)$ \\
\hline PSB-nonBF & $2(9)$ & $3(14)$ & 17 (77) \\
\hline \multicolumn{4}{|c|}{ Quantitative growth from the suspected VAP episodes without antibiotic pretreatment $(n=64)$} \\
\hline PSB & $38(59)$ & $3(5)$ & $23(36)$ \\
\hline BAL & $36(56)$ & $6(9)$ & $22(34)$ \\
\hline PSB-nonBF & $42(66)$ & $4(6)$ & $18(28)$ \\
\hline \multicolumn{4}{|c|}{ Quantitative growth from the suspected VAP episodes with antibiotic pretreatment $(n=24)$} \\
\hline PSB & $7(29)$ & $0(0)$ & $17(71)$ \\
\hline BAL & $6(25)$ & $1(4)$ & $17(71)$ \\
\hline PSB-nonBF & $8(33)$ & $2(8)$ & $14(58)$ \\
\hline
\end{tabular}

Percentage values are presented in parenthesis. VAP: ventilator-associated pneumonia; PSB: protected specimen brush; BAL: bronchoalveolar lavage; PSB-nonBF: PSB-non-fibroscopic.

BAL results in 23 out of 24 cases $(96 \%)$. PSB-nonBF agreed with the two bronchoscopic techniques combined in 21 out of 24 patients $(88 \%)$. The three diagnostic techniques had a much higher diagnostic yield in VAP episodes without antibiotic pretreatment.

Diagnostic value of the three techniques (only patients without antibiotic pretreatment included).

The sensitivity and specificity rates are shown in table 3. The sensitivity for suspected VAP episodes was not significantly different for PSB-BF vs BAL $(\mathrm{p}=0.72)$; PSB-BF $v s$ PSB-nonBF ( $\mathrm{p}=0.48$ ); and PSB-nonBF $v s$ BAL $(\mathrm{p}=0.28)$. The specificity also was not significantly different $(\mathrm{p}=0.28)$.

Results in pneumonia episodes with/without radiological involvement of the right lower lobe (RLL)

In episodes without RLL involvement $(n=10)$, significant growth of cultures was obtained with the three techniques in five patients $(50 \%)$. It must be noted that none of these patients were receiving any antibiotic treatment at the time of the sampling. The microbiological information yielded with these techniques was identical in the 10 cases $(100 \%)$.

Table 3. - Diagnostic value of the three techniques for the subgroup of episodes without antibiotic pretreatment $(n=64)$

\begin{tabular}{cccc}
\hline & PSB-BF & BAL & PSB-non BF \\
\hline Sensitivity (n=64) & & & \\
n & 38 & 36 & 42 \\
$\%$ & $59 \pm 6$ & $56 \pm 5$ & $66 \pm 6$ \\
$95 \%$ CI & $47-72$ & $46-67$ & $54-77$ \\
Specificity (n=22) & & & \\
n & 21 & 21 & 20 \\
$\%$ & 95 & 95 & 91 \\
Bp & $77.2-99.9$ & $77.2-99.9$ & $70.8-98.9$ \\
\hline
\end{tabular}

$\mathrm{n}$ : number of positives. $95 \%$ CI: $95 \%$ confidence interval; Bp: binomial probability. For further definitions see legend to table 2 .
In episodes with RLL involvement $(n=78)$, significant growth of cultures was obtained in 40 patients with PSB$\mathrm{BF}(51 \%)$; in 37 with BAL (47\%); and in 45 with PSBnonBF $(58 \%)$. No significant differences were observed between the technique used $\left(\chi^{2}=1.644 ; \mathrm{p}>0.1\right)$. In episodes with radiologically demonstrated RLL involvement, PSBBF and BAL agreed in 71 out of 78 cases (91\%); PSBnonBF and BAL in 64 out of $78(82 \%)$; PSB-BF and PSB-nonBF in 65 out of $78(83 \%)$; and PSB-nonBF and both bronchoscopic techniques in 65 out of 78 pneumonias $(83 \%)$. The data were similar to those observed in the total study population and those found in the population without RLL involvement (100\%) (Fisher's exact test).

\section{Secondary effects and associated iatrogenic response}

In our trial, both bronchoscopic studies as well as the PSB-nonBF were innocuous and also very well-tolerated by the patients. Minimal episodes of haemoptysis were observed that in no case required specific treatment.

\section{Discussion}

Correct diagnosis of ventilator-associated pneumonia requires a simple but safe technique, with a high degree of reliability. We investigated whether the Accu-Cath brush (Model PCC 215) met these characteristics, and found that this technique had an accuracy similar to that of the two bronchoscopic techniques, PSB-BF and BAL, commonly used in the diagnosis of VAP. Accu-Cath is a "blind" protected specimen bronchial brush (the tip is covered externally by plastic) with an inflatable balloon tip to avoid contamination of the samples, as described by other authors $[17,18]$.

One of the greatest problems with our study, as occurs with most investigations in VAP, is the correct diagnosis, if it is based exclusively on clinical-radiological criteria, because mechanically-ventilated patients frequently develop other conditions with a similar clinical picture [19-22], and techniques to be considered "gold standard" 
(lung biopsy and blood culture) have some limitations in their specificity [23]. As it is difficult to establish in practice a diagnosis of true VAP, most of the published studies have been made in patients with suspected VAP based on clinical criteria [9-14, 24-30], as we did in our study.

Some microorganisms, especially Haemophilus influenzae, Streptococcus pneumoniae, L. pneumophila and anaerobic bacteria, are very sensitive to saline transport media, and their concentrations decrease from 97 to $45 \%$ after one hour of exposure [31]. For this reason, we preferred a nonsaline medium $(1 \mathrm{~mL}$ of tryptone-soy bean (Tryptisoil@)), as was pointed out previously [16]. We have not considered the fungal growth, because it does not play an important role in nosocomial pneumonia except in neutropenic patients, not included in this study [32]. Whilst in other series, progressive dilutions of the original sample were cultured, in our study, only two quantities of known sample were cultured (0.1 and 0.01 $\mathrm{mL})$. We, therefore, may have undervalued some of the bacterial growth "limit" and hence obtained a lower sensitivity.

Our study showed that the diagnostic yield (sensitivity) and specificity of the PSB-nonBF in patients without antibiotic treatment was 66 and 91\%, respectively. Previous studies have reported similar values for various PSB-nonBF techniques. Using a simple protected brush, НА-PHAM et al. [27] obtained a sensitivity of $64.7 \%$ and a specificity of $93.5 \%$. With an analogous brush, JORDA et al. [13] identified the probable organisms involved in $80 \%$ of the pneumonias. TORRES et al. [10] obtained a sensitivity of $61 \%$ and a specificity of $100 \%$ by brushing through a Métras catheter. MiddLETon et al. [11] compared the recovery of bacteria using four techniques (PSB-BF, PSB-nonBF, BAL and semiquantitative bronchial aspirate). In four patients, using the Accu-Cath, they obtained a significant growth of 7 of the 9 organisms found by PSB-BF (considered by the authors as "almost-gold standard" technique). They also concluded that the bacterial recovery was similar using these four techniques. However, the small size of their sample (12 VAP patients) was an important limitation to reaching firm clinical conclusions [11]. If we consider all of the published reports, only the sensitivity obtained by LEAL-Noval et al. [12], which was only $35 \%$, is markedly different. The differences may be explained by the percentage of patients receiving antibiotic treatment at the time of sampling, and the variation in laboratory methodology.

There were no significant differences in the specificity of the PSB-nonBF between our series $(91 \%)$ and others $[10,13]$. The high specificity obtained by TORRES et al. [10] $(100 \%)$ is remarkable; and may be due to the inclusion of clinical and histological criteria.

Regarding our comparison of PSB-nonBF with procedures of recognized reliability (PSB-BF and BAL), we observed a very acceptable agreement between the information obtained by PSB-nonBF and that by the bronchoscopic techniques in the overall population and in each of the subgroups, as has also been shown in other series $[10-13,27]$.

The diagnostic yield from the PSB-BF in our study was similar to that obtained by several investigators $[10$, $18,24]$, but lower than that reported by others $[30,33$,
34], perhaps due to difference in culture methodology. The specificity obtained in our PSB-BF study was similar to that reported by many authors [10, 28-30, 35].

We found a diagnostic value for the BAL lower than that found in other studies $[6,7,21,24,36]$. Again, this could be due to the methodology employed in our laboratory (culture of two quantities of known sample), as done by other authors [36], since we did not apply the most frequently performed quantitative method of progressive dilutions [21, 22, 29]. Furthermore, we have used only $10 \mu \mathrm{L}$ inocula, and not both $10 \mu \mathrm{L}$ and 100 $\mu \mathrm{L}$ inocula [36], which invariably reduces the sensitivity. The specificity of BAL has been reported to vary from 69 to $71 \%$ [21, 29], even to 100\% [22, 36]. Again, this can be due to a difference in the cut-off points considered, from simple bacterial growth to $10^{4} \mathrm{cfu} \cdot \mathrm{mL}^{-1}$. The specificity obtained in our study was similar to that reported by those who considered $10^{4} \mathrm{cfu} \cdot \mathrm{mL}^{-1}$ as the cut-off point.

The agreement found between the two bronchoscopic techniques is remarkable (table 3) both in groups with and without antibiotic therapy. Our findings showed that the diagnostic yield was $10-52 \%$ greater in the group without antibiotic treatment, in agreement with other studies. Several authors reported a decrease in sensitivity and specificity with the use of antibiotics before sampling $[19,27]$. Therefore, it is recommended that antibiotics should not be administered $24 \mathrm{~h}$ prior to the use of these techniques in order to obtain maximum diagnostic yield [16].

An additional important issue is whether it is strictly necessary to perform the bronchial sampling in the involved lung area to obtain the greatest accuracy of protected brushing specimens. Our results could suggest that it is not necessary to sample the exact site which is radiologically involved. "Blind" brushing had the same reliability for the diagnosis of pneumonias with radiological involvement of the RLL as for those without. However, the small number of cases with no RLL involvement does not permit us to draw definitive conclusions in this respect. Furthermore, previous studies have reported contradictory findings $[6,12,28]$.

In conclusion, our findings suggest that the "blind" protected specimen brush has a similar diagnostic yield and specificity to bronchoscopic techniques. Sampling with the "blind" protected specimen brush (Accu-Cath) is a simple and reliable alternative. This could be interesting in those circumstances where standard bronchoscopic techniques are not available.

Acknowledgements: The authors wish to thank A. Torres for scientific assessment, and M. El-Ebiari for linguistic improvement of the manuscript.

\section{References}

1. Celis R, Torres A, Gatell JM, Almela M, RodriguezRoisin R, Agustí-Vidal A. Nosocomial pneumonia: a multivariate analysis of risk and prognosis. Chest 1988; 93: 318-324.

2. Banus Gasol S, Mateu Solá A, and Grupo de Estudio de la Neumonia Nosocomial adquirida en UCI. Epidemiology of the ICU-acquired pneumonia. Med Intensiva 1990; 14: 395-402. 
3. Jolliet Ph, Chevrolet JC. Bronchoscopy in the intensive care unit. Intensive Care Med 1992; 18: 160-169.

4. García Pachón E, Puzo C, Castella J. Complications of fiberoptic bronchoscopy. Arch Bronchoneumol 1993; 29: 153-157.

5. Craven DE, Daschner FD. Nosocomial pneumonia in the intubated patient: role of gastric colonization. Eur J Clin Microbiol Infect Dis 1989; 8: 40-50.

6. Rouby JJ, Rossignon MD, Nicolas MH, et al. A prospective study of protected bronchoalveolar lavage in the diagnosis of nosocomial pneumonia. Anesthesiology 1989; 71: 679-685.

7. Gaussorgues D, Piperno P, Bachmenn F, et al. Comparison of nonbronchoscopic bronchoalveolar lavage to open biopsy for the bacteriologic diagnosis of pulmonary infections in mechanically-ventilated patients. Intensive Care Med 1989; 15: 4-8.

8. Pipemo D, Gaussorgues P, Bachmann P, Jaboulay JM, Robert M. Diagnostic value of nonbronchoscopic bronchoalveolar lavage during mechanical ventilation. Chest 1988; 93: 223.

9. Zucker A, Pollack M, Katz R. Blind use of the double lumen plugged catheter for diagnosis of respiratory infections in ill patients. Crit Care Med 1984; 12: 867-870.

10. Torres A, Puig J, Rodriguez-Roisin R, Jimenez de Anta MT, Agustí-Vidal A. Diagnostic value of telescoping plugged catheters in mechanically-ventilated patients with bacterial pneumonia using Métras catheter. Am Rev Respir Dis 1988; 138: 117-120.

11. Middleton R, Broughton WA, Kirkpatrick MB. Comparison of four methods for assessing airway bacteriology in intubated, mechanically-ventilated patients. Am J Med Sci 1992; 304: 239-245.

12. Leal-Noval SR, Alfaro-Rodriguez E, Murillo-Cabeza F, Garnacho-Montero J, Rey-Perez J. Munoz-Sanchez MA. Diagnostic value of the blind brush in mechanicallyventilated patients with nosocomial pneumonia. Intensive Care Med 1992; 18: 410-414

13. Jorda R, Parras J, Reina J, Bergada J, Raurich JM. Diagnosis of nosocomial pneumonia in mechanicallyventilated patients by the blind protected telescoping catheter. Intensive Care Med 1993; 19: 377-382.

14. Johanson WG, Pierce AK, Sanford JP, Thomas GD. Nosocomial respiratory infections with Gram-negative bacilli: the significance of colonization of the respiratory tract. Ann Intern Med 1972; 77: 701-706.

15. Albertini R, Harrel J, Moser K. Hypoxemia during fiberoptic bronchoscopy. Chest 1974; 65: 117-122.

16. Meduri GU, Chastre J. The standardization of bronchoscopic techniques for ventilator-associated pneumonia. Chest 1992; 102 (5): 557S-564S.

17. Wimberley N, Faling LJ, Barbett JG. A fiberoptic bronchoscopy technique to obtain uncontaminated lower airway secretions for bacterial culture. Am Rev Respir Dis 1979; 119: 337-343.

18. Joshi JH, Wang KP, De Jongh C, Newman KA, Wiernik $\mathrm{P}$, Schimpff SC. A comparative evaluation of two fiberoptic bronchoscopy catheters: the plugged telescoping catheter versus the single sheathed nonplugged catheter. Am Rev Respir Dis 1982; 126: 860-863.

19. Fagon JY, Chastre J, Hance Aj, et al. Detection of nosocomial lung infection in ventilated patients: use of protected specimen brush and quantitative culture techniques in 147 patients. Am Rev Respir Dis 1988; 138: 110-116.

20. Fagon JY, Chastre J, Hance AL, Domart Y, Trouillet JL,
Gibert C. Evaluation of clinical judgment in the identification and treatment of nosocomial pneumonia in ventilated patients. Chest 1993; 103: 547-553.

21. Chastre J, Fagon JY, Soler P, et al. Diagnosis of nosocomial bacterial pneumonia in intubated patients undergoing ventilation: comparison of the usefulness of bronchoalveolar lavage and the protected specimen brush. Am J Med 1988; 85: 499-506.

22. Meduri GU, Beals DH, Maijub AG, Baselski V. Protected bronchoalveolar lavage: a new bronchoscopic technique to retrieve uncontaminated distal airway secretions. Am Rev Respir Dis 1991; 143: 855-864.

23. Meduri GU. Diagnosis and differential diagnosis of ventilator-associated pneumonia. Clin Chest Med 1995; 16: 61-93.

24. Guerra LF, Baughman RP. Use of bronchoalveolar lavage to diagnose bacterial pneumonia in mechanically-ventilated patients. Crit Care Med 1990; 18: 169-173.

25. Reina R, Jorda J. Quantification of bacterial isolations in the respiratory samples: value in the diagnosis of nosocomial pneumonia. Rev Esp Microbiol Clin 1990; 16: 19-23.

26. Xaubet A, Torres A. Valor del lavado broncoalveolar en el diagnóstico de las neumonias fúngicas y bacterianas. Med Clin (Barc) 1991; 96: 737-739.

27. Ha-Pham L, Brun-Buisson C, Legrand P, et al. Diagnosis of nosocomial pneumonia in mechanically ventilated patients: comparison of a plugged telescoping catheter with the protected specimen brush. Am Rev Respir Dis 1991; 143: 1055-1061.

28. Baughman R, Thorpe JE, Staneck J, Rashkin M, Frame $P$. Use of the protected specimen brush in patients with endotracheal or tracheostomy tubes. Chest 1987; 91: 233-236.

29. Torres A, Bellacasa JP, Xaubet A, et al. Diagnostic value of quantitative cultures of bronchoalveolar lavage and telescoping plugged catheters in mechanically-ventilated patients with bacterial pneumonia. Am Rev Respir Dis 1989; 140: 306-310.

30. De Castro FR, Violan JS, Capuz BL. Reliability of the bronchoscopic protected catheter brush in the diagnosis of pneumonia in mechanically-ventilated patients. Crit Care Med 1991; 19: 171-175.

31. Rein MF, Mandell GL. Bacterial killing by bacteriostatic saline solutions: potential for diagnostic error. $N$ Engl J Med 1973; 298: 784-795.

32. Bates JH, Campbell GD, Barron AL, et al. Microbial etiology of acute pneumonia in hospitalized patients. Chest 1992; 101: 1005-1012.

33. Lambert RS, Vereen LE, George RB. Comparison of tracheal aspirates and protected brush catheter specimens for identifying pathogenic bacteria in mechanically-ventilated patients. Am J Med Sci 1989; 297: 377-382.

34. Chastre J, Fagon JY, Soler P. Quantification of BAL cells containing intracellular bacteria rapidly identifies ventilated patients with nosocomial pneumonia. Chest 1989; 95: 190-192.

35. Cook J, Fitzgerald JM, Guyatt GH, Walter S. Evaluation of the protected brush catheter and bronchoalveolar lavage in the diagnosis of nosocomial pneumonia. J Intensive Care Med 1991; 6: 196-205.

36. Pugin J, Auckenthaler R, Mili N, Janssens JP, Lew PD, Suter PM. Diagnosis of ventilator-associated pneumonia by bacteriologic analysis of bronchoscopic and nonbronchoscopic "blind" bronchoalveolar lavage fluid. Am Rev Respir Dis 1991; 143: 1121-1129. 